\title{
Human Distributed Cognition from an Organism-in-its-Environment Perspective
}

\author{
Jaime F. Cárdenas-García, PhD, PE \\ Department of Mechanical Engineering \\ University of Maryland - Baltimore County \\ 1000 Hilltop Circle \\ Baltimore, MD 21250 \\ jfcg@umbc.edu \\ Timothy Ireland, $\mathrm{PhD}$ \\ Kent School of Architecture \\ Marlowe Building \\ University of Kent \\ Canterbury \\ Kent CT2 7NR \\ T.Ireland@kent.ac.uk
}

\begin{abstract}
The organism-in-its-environment is recognized as the basic unit of analysis when dealing with living beings. This paper seeks to define the fundamental implications of the concept of the organism-in-its-environment in terms of the biosemiotic concept of human distributed cognition. Human distributed cognition in a biosemiotic context is defined as the ability of a self-referencing organism-in-its-environment to interact with its environment to satisfy its physiological (internal and external) and social needs to survive and sustain itself. The ontogenetic development of the organism-in-its-environment serves as the backdrop to discover the implications of distributed cognition that have general applicability in organisms, but in this paper, are made relevant to human beings.
\end{abstract}

Keywords: Organism-in-its-Environment, Distributed Cognition, Ecology

\section{Introduction}

Hutchins (1995) coined the word "distributed cognition" in cognitive science, seeking “... to understand the organization of cognitive systems" by observing human activity "in the wild". Stressing that cognitive processes are distributed across members of a social group, he claimed, essentially, that the operation of the cognitive system involves coordination between internal and external (material or environmental) structures, and through time, in such a way that the products of earlier events can transform the nature of later events. Hutchins' efforts are directed at understanding how human activity develops in an "objective" and rational setting, in a cognitive science context. He uses the example of a ship, traversing into harbor but we propose the traditional London bus, operated by a driver and conductor, a more suitable biosemiotic example. A ship infers a certain scale, whereby the ship's crew communicate via devices enabling them to verbally convey individual circumstances and send/receive instructions. Additionally, whilst the crew work collectively they are supervised by their captain. With the London bus, driver and conductor work as a pair with no specific hierarchy and without verbal exchange. The driver is enclosed in his cab, whilst the conductor roams lower and upper decks. Their means of communication is a bell which the conductor chimes via a cord running the length of the decks. Given this cord is exposed it is open for passengers to use, which can lead to fuzzy instructions. The driver may thus respond to a signal not authored by the conductor, which could lead to the conductor overriding said signal. Consequently, driver and conductor may come to develop signals that passengers are not party to, involving not only the bell but the structure of the bus also. The conductor might ring the bell in a particular way or hit the side/inner walls to make a different sound, and the driver might make a particular maneuver. From the day of their coupling the pair will develop their means of exchange. The typical signals being a ring of the bell to instruct the driver to come to a stop and a double ring to start off, are sufficient to manage stopping and taking off again but the circumstances the pair encounter along their journey are beyond simply stopping and starting the bus to allow passengers on and off. The journey may also take the pair through different contexts and the traffic may 
fluctuate along their route day to day. Their route may change, or be varied due to external constraints. Consequently, the pair are required to mediate the managing of the bus between them as they traverse their route. It is unlikely this will start smoothly, as they must first become used to their confined tasks, the manner in which the other performs their particular task and the manner in which they do it. In time the pair will couple, mediating between themselves, their bus and the varied conditions they experience on their journey. Driver and conductor thus exemplify human distributed cognition through their coordination between material (bus) and external (passengers and context) structures, through indirect mediation which alters along a journey, and develops over time as the pair become acquainted, manage unpredictable journeys and changeable routes.

The approach followed in this paper seeks to redefine the concept of human distributed cognition from a biosemiotic perspective as the ability of a self-referencing organism-in-its-environment to interact with its environment to satisfy its physiological (internal and external) and social needs to survive and sustain itself. One aspect that makes human cognition distributed is the fact that humans unavoidably live in communities and social interactions are of paramount importance in their development. Human distributed cognition is inherent to human behavior and includes subjective and objective components. These objective and subjective components have considerable impact on human social interactions and result in the development of a complementary social space in a "Shared Universe", as will be shown later. We begin by considering the following characterization by Maturana and Varela (1987): “... cognition as an effective action that will enable a living being to continue its existence in a definite environment as it brings forth its world. Nothing more, nothing less." (Maturana \& Varela 1987: 29-30)

\section{The autopoietic homeostatic organism-in-its-environment}

The basic unit of analysis for consideration of human cognition is the human-organism-in-its-environment, and is depicted as shown in Figure 1. The process that allows the organism-in-its-environment to continue to survive and prosper is termed autopoiesis, or autopoietic organization, which refers to the capability of the organism for selfproduction but whose self-sufficiency is influenced by the subsuming environment niche (Maturana \& Varela 1987). It is also useful to note that even though the relationship of the organism with the environment is recursive (as noted by the opposite facing arrows), there is no implication that the relationship is symmetrical, rather, it is asymmetrical and this is denoted by using a segmented arrow as one of the double arrows. The asymmetrical nature of the relationship runs both ways. For example, the environment may act on the organism by way of natural events such as earthquakes or lightning bolts, that have no corresponding symmetrical response from the organism. Also, the organism may act on the environment by producing large scale environmental changes such as are common nowadays, setting off explosions to change the landscape, setting fires, or engaging in agriculture, which have no corresponding symmetrical response by the environment. However, this asymmetrical relation is not simply physical. The organismin-its-environment is an agential condition, informed through reciprocal exchange. Pentti Määttänen's (2010) analysis of this exchange points to a perception-action loop; emphasizing the spatial salience of this agential coupling. In action our modification of other things is more prominent than their reaction on us, as compared with perception, where the effect on us is overwhelmingly greater than our effect on that which is perceived. A loop is thus implied whereby action (output) is controlled with the help of received perceptual input. Perception is understood, in this thesis, simply as the distinction of a difference rather than cognitive: the capacity to distinguish a difference between different conditions (Bateson 1979). There is a strong similarity between Määttänen's analysis and Uexküll's concept of the functional cycle, albeit Määttänen stresses a coupling between organism and environment which is reciprocal as opposed to the prejudiced condition professed by Uexküll (1957).

The environmental sensing in this image is represented by the "Eye of Horus" where the symbols for smell, sight, hearing, taste and touch are depicted, with the implication that in general the human-organism is capable of distinguishing externally and internally generated stimuli. The depiction of the sense of touch also implies motility of the human-organism. There is no attempt here to assign priority to any of these senses, noting that all these senses act in concert to provide integrated sensing capability to the human-organism-in-its-environment. This also requires that a human-organism-in-its-environment acquire a capability for processing information. A fundamental understanding of the processing of information requires a basic definition of information that allows for clarity in its use and permits the use of said definition to build a useful corpus of knowledge. Bateson's dictum is a useful basis for this: "In fact what we mean by information - the elementary unit of information - is a difference which makes a difference..." 
(Bateson 1972: 321). Which, for the purposes of this discussion is amended to: "information is a difference which makes a difference to "somebody", (Hoffmeyer \& Emmeche 1991; Queiroz et al. 2008). One advantage of this definition is its generality and therefore applicability to all types of human-organism-in-its-environment interaction with information. Another noteworthy aspect of this definition is that it is a qualitative and subjective definition which relies on the human-organism-in-its-environment to make distinctions. This goes to the heart of the discussion on information, i.e., information may be viewed from a qualitative/subjective perspective and also from a quantitative/objective perspective.

Figure 1 illustrates how the ability to distinguish and process information leads to the development of ideation, or the capacity, of the human organism for the formation of ideas, thoughts or concepts, that allow for higher-level memory formation in dealing with its environment. When considering an ideating sensing human-organism-in-itsenvironment it is important to note that this marks the transition to the beginning of ideation as a way to gain greater access to the world by the sensing and acting human-organism-in-its-environment. Ideation is equivalent to having the capability of noticing differences, and requiring the development of the capacity for a higher level of memory formation, storage, accessing, utilization and knowledge construction.

Consideration of information, information processing and ideation as they relate to distributed cognition are not considered in detail here because the focus of this paper is on the general aspects of distributed cognition as a biosemiotic concept. But, quoting Bateson:

I suggest to you, now, that the word "idea," in its most elementary sense, is synonymous with "difference."' Kant, in the Critique of Judgment — if I understand him correctly — asserts that the most elementary aesthetic act is the selection of a fact. He argues that in a piece of chalk there are an infinite number of potential facts. The Ding an sich [thing as such], the piece of chalk, can never enter into communication or mental process because of this infinitude. The sensory receptors cannot accept it; they filter it out. What they do is to select certain facts out of the piece of chalk, which then become, in modern terminology, information. (Bateson 1972: 321)

This quote importantly points out: First, that the word "idea," in its most elementary sense, is synonymous with "difference"; Second, that a piece of chalk, which is generally white in color or encompasses "white light", takes the role of every object and represents an object with "... an infinite number of potential facts"; Third, because of this infinitude of facts which the "sensory receptors" of the organism are unable to process there is a need to "filter out" the excess facts, and to select only "certain facts" which are "information" with the underlying motivation of satisfaction of physiological needs.

\section{a. Emotions and the subjective behavior of the organism-in-its-environment}

The human-organism-in-its-environment represented in Figure 1 may be considered, in an unrealistic light, as existing by itself with no other living being around it. For a moment let us use this approach to examine such a humanorganism-in-its-environment by labeling the human-organism-in-its-environment as having a Personal/Subjective/Relative perspective, where these three words are taken in the context of the dictionary definition of these terms: Personal, i.e., pertaining to or characteristic of a person or self-conscious being: That is my personal belief ${ }^{1}$; Subjective, pertaining to or characteristic of an individual; personal; individual: a subjective evaluation ${ }^{2}$; and, Relative, existing or having its specific nature only by relation to something else; not absolute or independent: Happiness is relative ${ }^{3}$. There is also the connotation that in dealing with a Personal/Subjective/Relative perspective (PSR-perspective or view) (Cardenas-Garcia 2013) we are dealing with a qualitative assessment.

One of the main characteristics of a PSR-view is that it may be considered as an intrasubjective view, where this view is taken to be motivated by the satisfaction of physiological (internal and external) needs, where feelings and emotion play an important role. Examples of physiological needs as we interact with our environment are our needs

\footnotetext{
${ }^{1}$ Browsing entry "Personal" [Def. 1] in Dictionary.com.

2 Browsing entry "Subjective" [Def. 2] in Dictionary.com.

${ }^{3}$ Browsing entry "Relative" [Def. 3] in Dictionary.com.
} 
to eat, breathe, drink fluids and defecate, without which we would be unable to continue our homeostatic existence, and which if not satisfied is at the cost of our lives. Further, just to put these physiological needs in perspective, it is noteworthy that babies base their guidance to adults as to eating, defecating and heat/cold by crying loudly to be heard and helped in satisfying the associated physiological needs. This serves to highlight the fact that satisfaction of physiological needs has an emotive or affective component that can be unconscious (a baby just cries because of its unmet needs, not necessarily realizing the reason for its cries) and/or conscious (an adult feeling the pain of going hungry). Also, this concept of physiological needs is a dynamic concept and needs to be considered in the context of particular individuals. Consider that it is not the same to want to satisfy hunger while living a primitive lifestyle in the jungle as compared to doing so in New York city dependent on our socio-economic status. Additionally, as our experiences and tastes over our lifetime changes so do our physiological needs.

In summary, the PSR-view is inherent to the existence of the human-organism-in-its-environment when considering the idealized perspective of an isolated organism engaged in complementary interactions with its environment. This allows the discernment of the behavior of the organism-in-its-environment to be dominated by a PSR-view having a direct relationship to feelings and emotion. The PSR-view is inherent to our human existence and our first impressions in any new or existing situational setting. Our first reactions in any circumstance are circumscribed around feelings and emotions and revolve around satisfaction of physiological needs. This is the basis for our existence. Also, no one else has access to our PSR-view except as a result of the exteriorization of our feelings and/or emotions. This is comparable to the concept of the Umwelt put forward by Uexküll (1957), albeit Uexküll's Umwelt concept is species specific whereas what we articulate is individual specific.

\section{b. Reality and objective behavior of the organism-in-its-environment}

An aspect of behavior of the organism-in-its-environment that is also shown in Figure 1 are the asymmetrical complementary interactions between the organism and the environment. An environment is usually complicated by further comprising physical objects of a multiplicity of forms and texture apart from other living beings. In this process of an asymmetrical relationship, the organism encounters physical objects and other living beings that make it realize that some objective accounting has to take place if it is to continue the satisfaction of physiological needs. Physical objects or other living beings that need to be taken into account are ones which might have the capacity to cause pain/harm and pleasure/help to the organism-in-its-environment. One may hinder while the other may promote satisfaction of physiological needs. In either case, a more objective stance on the part of the organism-in-itsenvironment is needed to protect or promote such occurrences. This brings forth the need for the organism to develop the ability to project its particular viewpoint as to what it considers to be true about the workings of its environment. Some of these views might simply reflect the PSR-view of the organism-in-its-environment, while others might reflect its experience of pain/harm and pleasure/help in its interactions with its environment. When the PSR-view predominates, it becomes an instance where the organism-in-its-environment is able to believe anything it deems to be so, about itself and its surrounding environment, and may even mean that such viewpoints have either very small/large negative impact or smaller/greater positive impact on the well-being of the organism. On the other hand, when some actions lead to pain/harm the organism-in-its-environment takes notice and in so doing reflects that it has access, however small, to the beginnings of an Impersonal/Objective/Absolute perspective. Here again these three words are taken in the context of the dictionary definition of these terms: Impersonal, i.e., not personal; without reference or connection to a particular person: an impersonal remark ${ }^{4}$; Objective, not influenced by personal feelings, interpretations, or prejudice; based on facts; unbiased: an objective opinion ${ }^{5}$; and, Absolute, viewed independently; not comparative or relative; ultimate ${ }^{6}$. There is also the connotation that in dealing with an

${ }^{4}$ Browsing entry "Impersonal” [Def. 4] in Dictionary.com.

${ }^{5}$ Browsing entry "Objective” [Def. 5] in Dictionary.com.

${ }^{6}$ Browsing entry "Absolute" [Def. 6] in Dictionary.com. The use of "absolute" is made without losing sight of the term "relative", i.e., to emphasize the dynamic nature of the world around us. What is true today might not be true tomorrow. So, what we know today is relative to what we knew yesterday, but on an absolute scale we note that progress is being made. There is certainly an updating that is taking place. So, this progress might be viewed as relative and absolute at the same time, since both instances are concurrently present. 
Impersonal/Objective/Absolute perspective (IOA-perspective or view) (Cardenas-Garcia 2013) we are dealing with a quantitative assessment.

In short, when the PSR-view predominates, the qualitative/subjective nature of an organism-in-its-environment is implicit in Figure 1 above, because it is an image of a sole organism-in-its-environment. One overriding behavioral aspect of a single organism-in-its-environment is its qualitative/subjective nature. The qualitative/subjective nature may spring from immaturity or lack of experience, a self-centered attitude because of limited social interaction, or other environmental niche conditions that lead to temporal isolation of the organism. Sensory deprivation might be one such condition for adult prisoners in solitary confinement, which in extreme cases lead to hallucinations and odd behavior. This leads to a tendency for the human-organism-in-its-environment, as it sensorially engages with the spatial, temporal and meaningful confines of its environmental niche, to believe anything that is within the range of its integral, comprehensive and coherent experience. This range of experience can encompass the fantastical realm, but has to be confined (from its IOA perspective) within the limits of belief that allow the organism-in-its-environment to not compromise the integrity of its existence, i.e., the organism-in-its-environment should not engage in any action that leads to its demise. This may even take the form of a Principle of Existence for this lone organism-in-itsenvironment. This Principle has to be considered within the sensorial range that allows the organism-in-itsenvironment to explore the spatial, temporal and meaningful aspects of its environmental niche. These three dimensions of existence are accumulated concurrently as the organism-in-its-environment goes about its business of living.

While the limits of being of the organism-in-its-environment may exist in the fantastical realm, a dose of reality is always inherent in its actions due to its concurrent sensory experience which might include experiences that might have the potential of injury, such as sharp objects, tripping over objects, unsavory tastes, shrieking sounds, bad smells, curious predators, etc. which lend some objectivity to recurrent experiences, i.e., these may be considered the origin of an IOA-view. This ultimately means that the organism-in-its-environment is not encased without recourse in a PSRview or world, i.e., the organism-in-its-environment has the potential to become a being that lives a complementary PSR-view/IOA-view, these elements defining a relationship of complementary opposites, interdependence and uniqueness.

In summary, the experiences of the organism-in-its-environment include both qualitative/subjective and quantitative/objective aspects leading the organism-in-its-environment to discern the duality of its cognition: the organism-in-its-environment notices itself observing the environmental niche in which it dwells and in so doing takes notice of its own existence, irrespective of any interactions it might have with another similar organism-in-itsenvironment. The remaining question is whether such an organism-in-its-environment can access the full potential of the PSR-/IOA-views.

\section{The social organism-in-its-environment}

Figure 1 generalizes the conception of the environment to include anything and everything besides the organism. This figure emphasizes the fact that we exist as entities which interact with our surroundings by a limiting membrane. This limiting membrane separates our inner workings from our surroundings. Also, this membrane allows for observation, selection, manipulation and transformation of our inner space and outer surrounding space by means of our sense organs: touch, sight, sound, smell and taste. This helps us live as homeostatic beings, or beings capable of autopoiesis. Whether our sense organs provide us with full or even erroneous information about our inner workings and surroundings is irrelevant in order to know that this is our only window to our internal and external universe. Whether our experience, brought about by the use of our senses, is qualified as subjective or objective is irrelevant to being organs of information gathering of which we may or may not be aware. It is also possible that we might be unaware of the process of observing, selecting, manipulating and transforming our inner and outer universes, and their eventual impact on us as a result of our activities.

An initial description of Figure 1 portrays us as an organism-in-its-environment with a PSR-view of reality, whatever that might be; if we can imagine ourselves as beings living alone in our environment. This PSR-view of reality, as mentioned previously, is also tinged with an IOA-view of reality in complementary unity, even when considering this unrealistic solitary state of existence of the organism-in-its-environment. This complementary PSR- 
view/IOA-view negates and obviates a solipsistic view of the organism-in-its-environment such as the one expressed by Maturana \& Varela, which does not seem to consider the effect of social and other interactions with the environment that are capable of generating an IOA-view:

No description of an absolute reality is possible. Such a description would require an interaction with the absolute to be described, but the representation which would arise from such an interaction would necessarily be determined by the autopoietic organization of the observer, not by the deforming agent; hence, the cognitive reality that it would generate would unavoidably be relative to the knower. (Maturana \& Varela 1980: 121)

As human beings, we are faced with the reality that we are born from the procreative interaction of two human beings. We are parented and live as part of a community. This more realistic variation is depicted in Figure 2 , for ideating and sensing human-organisms-in-their-environment, where another human-organism-in-its-environment shown on the right side of the figure (organism 2) is added and may be regarded by the human-organism-in-itsenvironment to the left of the figure (organism 1) as just another part of the environment. Indeed, there is a reciprocal appraisal on the part of both human-organisms-in-their-environment as to what constitutes their own environment. Every type of social interaction between two human-organisms-in-their-environment may be characterized in this fashion. Consideration of social interaction is oftentimes neglected but needs to be recognized as the basis of distributed cognition being integral and intrinsic for a human-organism-in-its-environment ${ }^{7}$.

\section{Distributed cognition}

Previously we defined distributed cognition as the ability of a self-referencing organism-in-its-environment to interact with its environment to satisfy its physiological (internal and external) and social needs to survive and sustain itself. This definition applies both, more generally to Figure 1 and, more particularly to the more realistic representation of Figure 2 where social interaction is made explicit, and is inherent and integral to a social humanorganism-in-its-environment.

The human-organism-in-its-environment of Figure 1 was characterized as capable of exhibiting a complementary PSR-view/IOA-view, where its own preferences and viewpoint takes center stage, whilst capable of gaining access to greater objectivity: avoiding solipsism. Now in Figure 2 we are dealing with two such human-organisms-in-theirenvironment, one of which holds a Personal/Subjective/Relative perspective or (PSR view) 1 , shown by the circle to the right of organism 1, which is in all probability different from that of the other human-organism-in-its-environment with a (PSR view) $)_{2}$, shown by the circle to the left of organism 2. Above we noted that the complementary recursive interactions between the organism and its environment are asymmetrical as designated by the double arrows: one solid, the other segmented. This is also true of the complementary social interactions between two human-organismsin-their-environment as shown in Figure 2. Recall that complementary interactions require contradictory opposites that are interdependent and unique, and the possibilities for social interaction run the gamut from peaceful to conflictive. The complementary social interactions between these two human-organisms-in-their-environment and the identification of the applicable basic principles needs consideration. Let us then define and explore the complementary social space that results from complementary social interactions.

\section{Development of a complementary social space}

Complementary asymmetrical interactions are the natural condition of human existence as organisms-in-ourenvironment. This results in a multiplicity of repeated and consistent long-term interactions with other living beings and inorganic matter of every kind, which allow for multifarious experiences impinging on all of our senses, constrained by place and time. Limiting our interest to complementary social interactions between organisms-in-theirenvironment it is useful to define the existence of a resulting complementary social space, shown in Figure 2 by the designated intersection between the two circles designated as (PSR view) ${ }_{1}$ and (PSR view) 2 , respectively, and labelled as "Shared Universe", and further identified as (IOA view). What is being recognized is that if social interactions are established between two organisms-in-their-environment the complementary PSR-view/IOA-view of each needs to

${ }^{7}$ For an enactive perspective of social interaction see (De Jaegher \& Di Paolo 2007; Fuchs \& De Jaegher 2008; Froese \& Di Paolo 2009) 
be reduced by the "Shared Universe" of both. This has at least three possibilities: a) The "Shared Universe" includes only PSR-view elements; b) The "Shared Universe" includes only IOA-view elements; and, c) The "Shared Universe" includes both PSR-view and IOA-view elements. An example of this last notion of "Shared Universe" is that which is created by the complementary interactions of mother/caretaker and child, requiring constant and recursive interactions including suckling, touching and caring in an asymmetrical exchange. In this particular instance, the dependency of the child on the mother/caretaker might imply that the "Shared Universe" mainly contains PSR-view elements on the part of the child (such as crying because of unmet needs: i.e., hunger), which are accommodated by the mother/caretaker (who can sympathize: i.e., experience of hunger and realization of said child's physiological needs); thereby incorporating both PSR-view and IOA-view elements.

Each individual may have her own PSR/IOA universe but the need for communication and the forming of communities requires the building of a "Shared Universe" which is consensual in nature. This sharing results in the forming of consensual bonds between individuals which may be widely shared by the community. In this process of sharing, the PSR/IOA universe of each individual requires its reduction by the amount of the "Shared Universe". This is a similar view as that of Bitbol when referring to the "Neurophenomenology" of Varela (1996 and 1997),

The basis of his approach was the remark according to which any third person, objective, description, arises as an invariant focus for a community of embodied, situated, subjects endowed with conscious experience in the first place. This remark is usually either overlooked (by those philosophers who think invariance is only our way to discover a reality behind the "superficial" situated appearances), or overrated (by those philosophers who use it as a weapon against any claim of knowledge). The two former attitudes yield a systematical bias towards conscious experience. (Bitbol 2002: 181)

This means that the PSR/IOA beliefs that each individual holds are modified in a historical process that requires modification of individual PSR/IOA views in pursuit of common ground between individuals, a process that also has to meet the criteria that such common beliefs cannot be in conflict with the survival and sustenance of the group. This may even take the form of a Group Principle of Existence for these organisms-in-their-environment engaged in complementary social interactions. That the social organism-in-its-environment may not engage in any behavior nor hold any belief about itself or its environmental niche that would lead to the demise of the group.

Another example where the "Shared Universe" is mainly composed of PSR-view elements is that related to religion or belief systems. While an example that is composed solely of IOA-view elements is probably difficult to find since as mentioned above, we are beings whose PSR-view is basic to our existence. Science and the scientific method is an example where the "Shared Universe" is constructed with the view that the IOA-view becomes predominant, but it is a view which cannot but evolve from a PSR-view, and this is worth examining in greater detail.

\section{Science and the scientific method}

Scientists generally promote the perspective that their efforts relate to the objective aspects of our existence. Science is thought to be mainly objective, i.e., that in order for something to be scientific it has to be obtained by following the scientific method, based on observation and theorizing, to pose a hypothesis which is then tested using a repeatable protocol to yield results that are scrutinized by a community of scientists to verify the validity of the hypothesis, thus yielding additional experience leading to a new cycle of creative hypothesizing in a never ending recursive cycle. This defines a process that is repeatable by anyone with the required skills, and which allows greater and greater approximation to reality by the never-ending and continuous application of the scientific method. This makes the scientific enterprise historical in nature, i.e., what came earlier has the possibility of impacting what comes after. The scientific results that are currently accepted may be superseded by newly generated results and insights that are further corroborated in this endless process. A historical process that is methodical may be said to yield results that change over time, i.e., at any point in time these results may be measured on an absolute temporal basis but realizing that they in all probability will lead to new insights and changes. So, any particular scientific result is always in the process of changing or becoming something new and better. So, from a complementary perspective, the scientific enterprise is always evolving toward greater knowledge. So, it can be argued that scientific advancement has relative and absolute aspects. 
But scientists have to undergo a process of socialization into science. No one is born a scientist, but rather human beings engage in an educational process, theoretical and empirical, that results in human beings that graduate into being scientists. In this regard, a question that requires an answer is: How does a subjective being become an objective being? Thus, science and the scientific method may be examined as a process that encompasses the "Shared Universe" perspective that requires inclusion of PSR-view and IOA-view elements, implying a combined First-Person/ThirdPerson Perspective. This distinction of equating the PSR-view with the First-Person perspective and correspondingly the IOA-view with the Third-Person perspective applies only to science and the scientific method. The implication is that the PSR-view and IOA-view are more general than the First-Person and Third-Person perspective, respectively. Ontogenetically it may be argued that human beings begin their life more on the PSR-view side of the equation evidencing a First-Person Perspective. As they mature, the IOA-view acquires greater importance (especially for someone with a scientific bent), and the Third-Person Perspective gains importance. Eventually, no matter how reputable a scientist becomes it is impossible to say conclusively that every aspect of her professional practice concerns only the Third-Person Perspective. No scientist makes discoveries without being motivated subjectively to make those discoveries from a First-Person Perspective, yet no scientist makes discoveries that he can defend and gain recognition without a Third-Person Perspective. As a result, it is unavoidable that a scientist has a combined First-Person/ThirdPerson Perspective that corresponds to a combined PSR-view and IOA-view. In other words, the use of the scientific method starts as an individual endeavor, in which individual participants engage in scientific inquiries. Those individual inquiries only acquire currency when confronted with the collective efforts of countless individuals that constitute the scientific enterprise. Reality involves a PSR-view in the never ending short-term, and reality also simultaneously involves an IOA-view in the never ending long-term. In short, PSR-views are primary but incomplete without IOA-views, and the centrality of human beings cannot be dismissed even in scientific discourse.

\section{Alienation and fetishism of complementary consensual space}

It is well known and not unusual that we sometimes ascribe supernatural properties or even personality to objects. This might be the case for a rabbit's foot, a crucifix or even a crystal with medicinal/spiritual properties. To illustrate how this process may come about, let us consider the interaction of organism 1 with the environment (shown in the lower left of organism 1 and also to the left of the vertical double arrows), using similar circles as described above for the social interaction between organism 1 and organism 2, as shown in Figure 2. A similar set of circles is equally visible between organism 2 and the environment (shown in the lower right of organism 2 and also to the right of the vertical double arrows). The purpose of these circles is to describe how the human organism is able to create a "Shared Universe" with the environment, which in this assessment we are going to limit to that of only nonliving matter in the environment. Whatever conclusions we are able to reach relative to nonliving matter will be applicable to living matter in the environment with very little modification.

More specifically, the circle associated with organism 1 is labelled (PSR view) ${ }_{1}$ and the circle associated with the

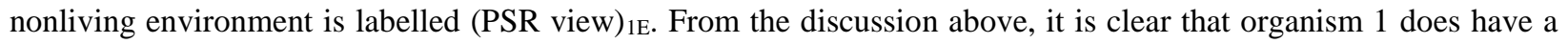
PSR-view/IOA-view, but what is not clear is that the nonliving environment is capable of even a PSR-view. This process may be explained by considering the following quote taken from Silva in making reference to Berger \& Pullberg (1965):

Approaching reification from a phenomenological and cognitive perspective (the way phenomena appear to human consciousness and become cognized), Berger and Pullberg (1965) describe reification as the final stage in a four-stage process. They call the first stage objectivation because, as they put it, we humans embody our intentionality in the products we create, from material objects to such abstractions as names. The second stage, objectification, happens at the point in the process of objectivation in which we distance ourselves from our products and take cognizance of them as objects of consciousness. Now, not only do we objectivate, but we also recognize our products as such. Later, in the stage of alienation, as the distance between us and our products continues to grow, we forget that we ourselves created those products, which now appear to us in consciousness as 'alien facticities'. The fourth and last stage, reification, takes place when we begin to apprehend those things as not only alien but also real. (Silva 2013: 84) 
The approach that we take is one that begins with organism 1 exhibiting a PSR-view/IOA-view or (PSR view) 1 of reality in which we engage in objectivation and objectification, e.g., of a rabbit's foot. We then engage in a process of alienation where we no longer recognize that we have had a role in not only making the rabbit's foot but in ascribing meaning to the reason for its creation (as a source of luck for its owner), to the reification of the rabbit's foot where it is alien to us and has its own reasons for its existence. In this stage of alienation is where we now ascribe a (PSR) ${ }_{1 E^{-}}$ view to the object and in so doing we are now in a position to have a "Shared Universe" with the rabbit's foot. This shared universe reiterates the perception-action loop mentioned earlier, whereby the effect of the rabbit's foot on the individual (perception of its merits) affects certain action, which reinforces one's view of the rabbit's foot. Of course, this can apply to any object with which we might enter into contact, and over time engage in this process that ends in alienation followed by fetishism, or the ability of a non-living object to acquire supernatural significance.

\section{Summary and Conclusions}

Recognizing that the basic unit of analysis in a biosemiotic world is the organism-in-its-environment, we begin the ontogenetic exploration of the concept of distributed cognition for humans. Distributed cognition is defined as the ability of a self-referencing human-organism-in-its-environment to interact with its environment to satisfy its physiological (internal and external) and social needs to survive and sustain itself. This definition encompasses consideration of the complementary social interactions between human-organisms-in-their-environment, as well as the more traditionally regarded divisions of the emotive and cognitive domains which are inherently contained in examining a human-organism-in-its-environment.

The approach followed in this paper seeks to redefine from a biosemiotic perspective the concept of distributed cognition in an effort to discover that it is a concept: inherent to human-organism-in-its-environment behavior; that includes emotive (subjective) and cognitive (objective) as inherent aspects to its expression in human-organisms; and, which has considerable impact on human social interactions which results in the development of a complementary social space in a "Shared Universe". Following this same approach in interaction with non-living objects reveals that it is possible to engage in alienation followed by fetishism when these non-living objects acquire supernatural significance.

\section{Acknowledgements}

The authors would like to acknowledge the reviewers for their comments and suggestions, which have helped to significantly improve the content of this paper.

\section{References}

Bateson, G. (1972). Steps to an Ecology of Mind. (Repr. 1987) Northvale, New Jersey: Jason Aronson Inc.

Bateson, G. (1979). Mind and Nature: A Necessary Unity. New York: E. P. Dutton.

Berger , P., \& Pullberg, S. (1965). Reification and the sociological critique of consciousness. History and Theory, 4(2), $196-211$.

Bitbol, M. (2002). Science as if situation mattered. Phenomenology and the Cognitive Sciences, 1(2), 181-224.

Cardenas-Garcia, J. F. (2013). Distributed Cognition: An Ectoderm-Centric Perspective. Biosemiotics, 6(3), 337-350.

De Jaegher, H., \& Di Paolo, E. (2007). Participatory Sense-Making An Enactive Approach to Social Cognition. Phenomenology and the Cognitive Sciences, 6(4), 485-507.

Dictionary.com - definitions. Online dictionary in http://www.dictionary.com/. Accessed July 9, 2015.

Froese, T., \& Di Paolo, E. A. (2009). Sociality and the life-mind continuity thesis. Phenomenology and the Cognitive Sciences, 8(4), 439-463.

Fuchs, T., \& De Jaegher, H. (2009). Enactive intersubjectivity: Participatory sense-making and mutual incorporation. Phenomenology and the Cognitive Sciences, 8(4), 465-486.

Hutchins, E. (1995). Cognition in the Wild. Cambridge, MA: MIT Press.

Hoffmeyer, J. \& Emmeche, C. (1991). CodeDuality and the Semiotics of Nature. In Myrdene Anderson, \& Floyd Merrell (Eds.), On Semiotic Modeling (pp. 117-166). Berlin and New York: Mouton de Gruyter. 
416

417

418

419

420

421

422

423

424

425

426

427

428

429

430

Määttänen, P. (2010). Habits as Vehicles of Cognition. Ideas in Action: Proceedings of the Applying Peirce Conference, Nordic Studies in Pragmatism 1. Helsinki: Nordic Pragmatism Network. M. Bergman, S. Paavola, A.-V.Pietarinen and H. Rydenfelt (eds.), 201-210.

Maturana, H., \& Varela, F. (1980). Autopoiesis and Cognition - The Realization of the Living. Dordrecht, Holland: D. Reidel Publishing Company.

Maturana, H., \& Varela, F. J. (1987). The Tree of Knowledge: The Biological Roots of Human Understanding. Boston, MA: Shambhala Publications, Inc.

Queiroz, J., Emmeche, C., \& El-Hani, C. N. (2008). A Peircean Approach to 'Information' and its Relationship with Bateson's and Jablonka's Ideas. The American Journal of Semiotics, 24(1-3), 75-94.

Silva, S. (2013). Reification and Fetishism: Processes of Transformation. Theory, Culture \& Society, 30(1), 79-98.

Uexküll, Jakob von . (1957). A stroll through the worlds of animals and men. Instinctive Behavior: The Development of a Modern Concept, Claire H. Schiller (ed. and trans.). New York: International Universities Press, 5-80.

Varela, F. J. (1996). Neurophenomenology. Journal of Consciousness, 3, 330-349.

Varela, F. J. (1997). The Naturalization of Phenomenology as the Transcendence of Nature: Searching for generative mutual constraints. Alter: Revue de Phénoménologie, 5, 355-385. 


\section{List of Figures}

Figure 1 - The sensing and ideating autopoietic homeostatic organism-in-its-environment Figure 2 - The social sensing and ideating autopoietic homeostatic organism-in-its-environment 


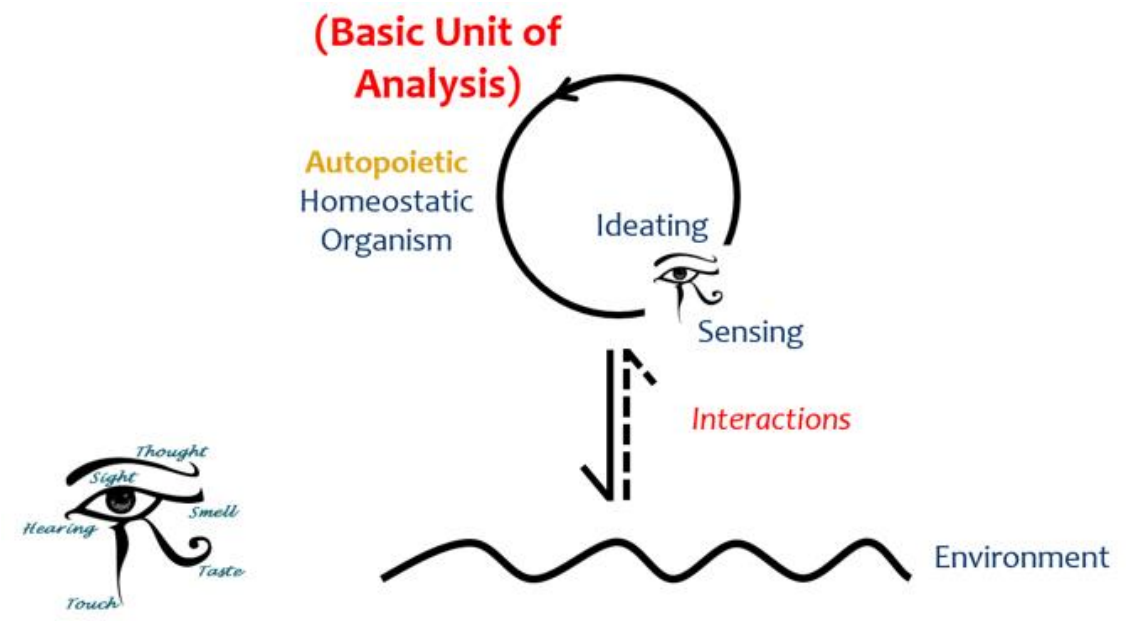

Figure 1 


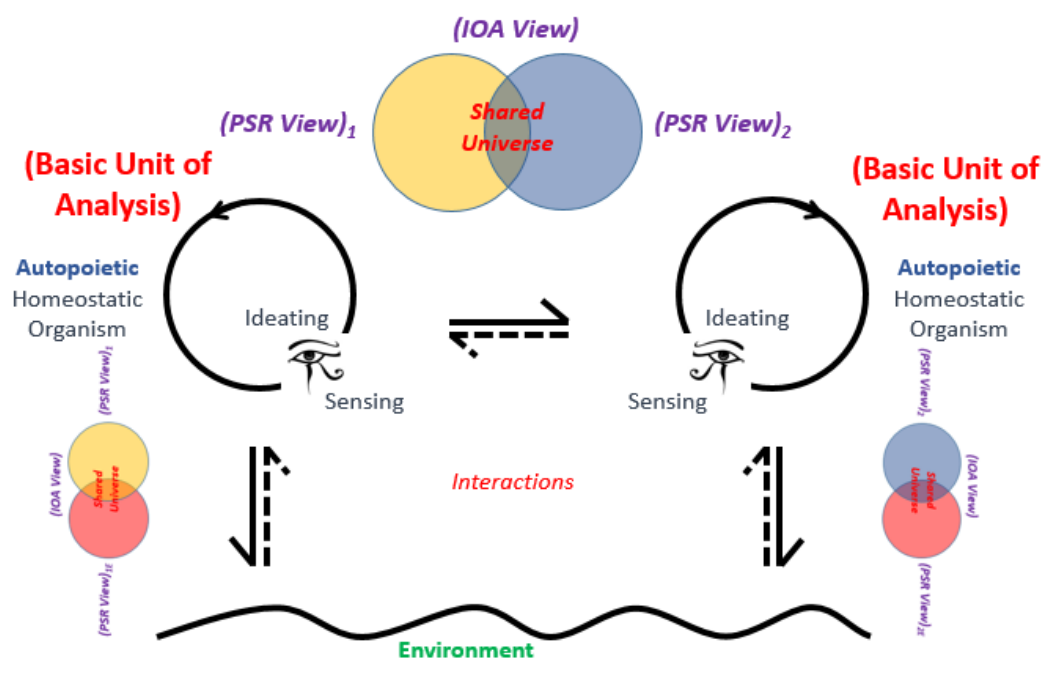

Figure 2 